\title{
Regulation of the cholesterol biosynthetic pathway and its integration with fatty acid biosynthesis in the oleaginous microalga Nannochloropsis oceanica
}

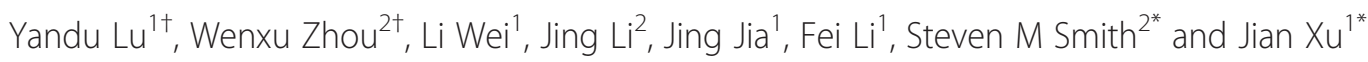

\begin{abstract}
Background: Sterols are vital structural and regulatory components in eukaryotic cells; however, their biosynthetic pathways and functional roles in microalgae remain poorly understood.

Results: In the oleaginous microalga Nannochloropsis oceanica, the sterol biosynthetic pathway produces phytosterols as minor products and cholesterol as the major product. The evidence together with their deduced biosynthetic pathways suggests that $\mathrm{N}$. oceanica exhibits features of both higher plants and mammals. Temporal tracking of sterol profiles and sterol-biosynthetic transcripts in response to changes in light intensity and nitrogen supply reveal that sterols play roles in cell proliferation, chloroplast differentiation, and photosynthesis. Furthermore, the dynamics of fatty acid (FA) and FA-biosynthetic transcripts upon chemical inhibitor-induced sterol depletion reveal possible co-regulation of sterol production and FA synthesis, in that the squalene epoxidase inhibitor terbinafine reduces sterol content yet significantly elevates free FA production. Thus, a feedback regulation of sterol and FA homeostasis is proposed, with the 1-deoxy-D-xylulose 5-phosphate synthase (DXS, the committed enzyme in isoprenoid and sterol biosynthesis) gene potentially subject to feedback regulation by sterols.

Conclusion: These findings reveal features of sterol function and biosynthesis in microalgae and suggest new genetic engineering or chemical biology approaches for enhanced oil production in microalgae.
\end{abstract}

Keywords: Cholesterol biosynthetic pathway, Fatty acid biosynthesis, Feedback regulation, Nannochloropsis

\section{Background}

Sterols are vital components of all eukaryotic cells [1]. In higher plants, they play a structural role in cell viability, embryogenesis, pattern formation, cell division, chloroplast biogenesis, and modulation of activity and distribution of membrane-bound proteins such as enzymes and receptors [2,3]. In addition, sterols are precursors for many signaling molecules that regulate growth and development in plants and animals, such as insect ecdysteroid

\footnotetext{
* Correspondence: steven.smith@uwa.edu.au; xujian@qibebt.ac.cn ${ }^{\dagger}$ Equal contributors

${ }^{2}$ Australian Research Council, Centre of Excellence in Plant Energy Biology, The University of Western Australia, 35 Stirling Highway, Crawley, WA 6009, Australia

'Single-Cell Center, CAS Key Laboratory of Biofuels and Shandong Key Laboratory of Energy Genetics, Qingdao Institute of BioEnergy and Bioprocess Technology, Chinese Academy of Sciences, Qingdao, Shandong 266101, China
}

molting hormones [4], mammalian steroid hormones [5], and plant brassinosteroid (BR) hormones [6].

Sterols belong to a class of isoprenoids derived from isopentenyl pyrophosphate (IPP), a universal precursor of isoprenoids. In animals and fungi, the cytoplasmic mevalonic acid (MVA) pathway is the only route for biosynthesis of IPP, the building block for lanosterol, which is then metabolized into cholesterol in animals and ergosterol in fungi [7]. In higher plants, IPP can be derived via either the MVA pathway or the plastidial 1-deoxyxylulose 5-phosphate or methylerythritol phosphate (MEP) pathway, despite the former being the main contributor to sterol biosynthesis [8,9]. In Arabidopsis, sterol biosynthetic mutants can be classified into two distinct groups: BRindependent mutants, which are defective in genes in the pathway from cycloartenol to 24-methylenelophenol [10],

\section{Biomed Central}

(C) 2014 Lu et al.; licensee BioMed Central Ltd. This is an Open Access article distributed under the terms of the Creative Commons Attribution License (http://creativecommons.org/licenses/by/4.0), which permits unrestricted use, distribution, and reproduction in any medium, provided the original work is properly credited. The Creative Commons Public Domain Dedication waiver (http://creativecommons.org/publicdomain/zero/1.0/) applies to the data made available in this article, unless otherwise stated. 
and BR-dependent mutants, which are defective in genes at the latter part of the sterol biosynthetic pathway (from 24-methylenelophenol to campesterol, which is considered as the precursor for BRs) [11]. Besides serving as a BR precursor, sterols appear to play distinct signaling functions during plant development, since phenotypes of sterol biosynthetic mutants cannot be rescued by addition of exogenous BR [12].

Co-regulation of sterol synthesis and fatty acid (FA) production is essential for maintaining the biosynthesisversus-turnover balance of membranes during cellular growth [13]. In animals, sterols and FAs are coordinately regulated by a feedback system mediated by a conserved family of transcription factors called sterol regulatory element binding proteins (SREBPs), which controls a cascade of biosynthetic enzymes for endogenous cholesterol, FA, triacylglycerol (TAG), and phospholipid $[14,15]$. Activated SREBP binds to sterol response elements in the promoter and/or enhancer regions of target genes and induces transcription of at least 30 cholesterol- and lipidsynthesis genes (particularly those encoding rate-limiting enzymes, such as hydroxy-methyl-glutaryl-CoA reductase, HMGR and type I FA synthase, FAS) [16]. Manipulation of this regulatory cascade in transgenic mice resulted in 6and 22-fold increases in cholesterol content and TAG content, respectively, and consequentially massive fatty livers [17].

Microalgae are promising feedstock for sustainable and scalable production of biofuel [18]; however, few microalgal strains found in nature are endowed with the wide array of traits demanded by a large-scale and economically competitive production scheme [19]. It is therefore urgent to identify molecules and mechanisms that regulate microalgal growth, development, and stress responses for strain improvement. Sterols in microalgae display enormous diversity due to the high degree of phylogenetic heterogeneity, the vast number of genera, and the long evolutionary distance among many of them $[20,21]$. It has long been shown that in microalgae sterol composition varies upon changes in growth stage, light spectrum, or temperature, suggesting important yet largely unknown roles of sterols [22]. Therefore, manipulation of sterol biosynthesis and regulation offers potential for engineering lipid production. Exploration of the sterol-dependent lipogenesis regulatory mechanism in microalgae might provide novel strategies and targets for enhanced lipid production in microalgae. However, little is known about the roles, biosynthesis, and regulation of sterols in microalgae and in particular, whether and how sterol and FA metabolism is coregulated.

Nannochloropsis spp. are a genus of unicellular photosynthetic microalgae belonging to the heterokonts. They are distributed widely in the marine environment as well as in fresh and brackish waters. These algae are of industrial interest because they grow rapidly and can synthesize large amounts of TAG and high-value polyunsaturated FA (for example, eicosapentaenoic acid) [23]. The genomes of multiple species of oleaginous Nannochloropsis spp. have been sequenced and annotated [23-27]. Employing an oleaginous industrial microalga $N$. oceanica IMET1 as a model, this study has aimed to determine the sterol composition and biosynthetic pathway in microalgae, to investigate the role of sterol biosynthesis in photosynthesis and growth, to study the influence of light and nitrogen supply, and to probe the effects of sterol levels on FA accumulation. Our findings expand the understanding of sterol function in microalgae and should assist rational genetic or process engineering for microalgae-based production of biofuels or other value-added bioproducts.

\section{Results}

N. oceanica sterol biosynthetic pathway shares features in structure and sterol profiles with those of animals and plants

Among different organisms, the core sterol biosynthetic pathway consists of a common set of enzymes that exhibit strong conservation in amino acid sequences; however the pathway architecture and substrate specificity can vary significantly [1]. In silico reconstruction and comparison of sterol biosynthetic pathways among 12 selected algal species revealed intriguing structural features of the $N$. oceanica pathway, which include characteristics from both higher plants and animals (Figure 1 and Additional file 1).

The sterol synthetic pathway of $N$. oceanica includes higher plant-like features. Higher plants have two sterol methyltransferase (SMT) enzymes that use different substrates to give either methylated (SMT1) or ethylated (SMT2) phytosterols. In the $N$. oceanica genome, two candidate genes encoding SMT were identified, which resemble those of higher plants in primary sequence. In contrast, the diatom Phaeodactylum tricornutum and several green algae including Chlamydomonas reinhardtii, Chlorella variabilis NC64A, Coccomyxa subellipsoidea C-169, and Volvox carteri have a single candidate gene encoding SMT (Figure 1, see Additional file 2: Figure S1 for the phylogenetic tree of the sampled species) that potentially catalyzes successive methylation reactions to give methylated and ethylated products.

Features that are shared with animals were also present in the sterol synthetic pathway of $N$. oceanica. In the sampled microalgae, the key enzyme catalyzing sterol side chain reduction is different from that of Arabidopsis and higher plants in general. In higher plants, the enzyme, namely sterol 24(28) isomerase-reductase, is encoded by the DWF1 gene in Arabidopsis and performs dual functions. It catalyzes C-24(28) double bond isomerization to 


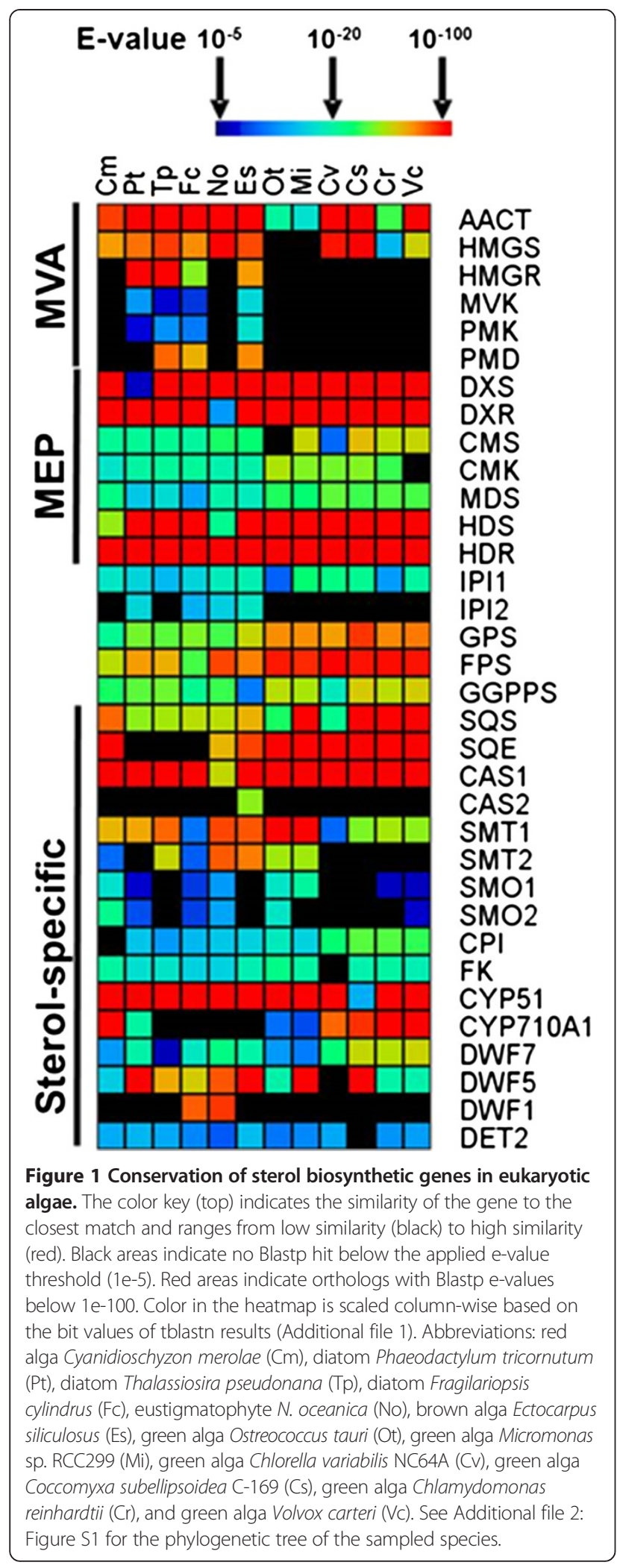

form a 24(25) double bond, followed by reduction of the 24(25) double bond. In animals and yeast, the equivalent enzymes are 24-dehydrocholesterol reductase (DHCR24) and sterol C-24(28) reductase (ERG4), which only catalyze the reduction reaction. DWF1 or DHCR24 orthologs have not been found in algae except in N. oceanica and the diatom Fragilariopsis cylindrus. Amino acid sequence analysis indicates that $N$. oceanica sterol 24(25) reductase is clustered with that of choanoflagellates (the closest living unicellular relatives of animals [28]) and has greater similarity to animal DHCR24 than to higher plant DWF1 (Additional file 2: Figure S2). The evidence based on DWF1/DHCR24 therefore suggests features of an animaltype sterol biosynthetic pathway.

To test these predicted features of the sterol biosynthetic pathway, we characterized the chemical profile of sterols in $N$. oceanica IMET1, which unveiled an animal-like composition of sterols. In $N$. oceanica, five sterols were identified (sterol structures indicated as bold numbers in Additional file 2: Figure S3 and in the following text). Cholesterol (2) is the most abundant sterol, comprising $70 \%$ to $75 \%$ of the total (Table 1 ). The remaining sterols are fucosterol (11), isofucosterol (13), 24-methylcholesta-5, 25(27)-dienol (10), and 24methylenecholesterol (7) (Additional file 2: Figure S3). In $N$. oceanica, sterols with a C-22 double bond are not found, supporting the absence of CYP710A (a C-22 desaturase; Figure 1). Although a protein (g4528) with similarity to CYP710A was present, its primary sequence is closer to that of CYP51 than to CYP710A (Additional file 1). The lack of CYP710A is a typical feature of animals, as higher plant genomes usually encode highly conserved CYP710A. In addition, the side-chain double bond formed by SMTs is retained, supporting the presence of an animal-type DHCR24 (Additional file 2: Figure S2). This is further supported by the accumulation of a large amount of cholesterol in $N$. oceanica (Table 1), which is the sole sterol in animals. On the other hand, only a minor amount of the phytosterols, which are the dominant forms of sterols in higher plants, was found in $N$. oceanica (Table 1). Therefore, $N$. oceanica sterol profiles exhibit features of both animals and higher plants.

Furthermore, we adopted a chemical biology approach to probe the architecture of the sterol biosynthetic pathway, in which $N$. oceanica was treated with a series of isoprenoid and sterol biosynthetic inhibitors (SBIs). (See Figure 2 for the target enzymes and Additional file 2: Figure S4 for the inhibition ratios.) The phenotypes of sterol biosynthetic mutants can be mimicked by the application of specific chemical inhibitors, which are powerful tools for elucidating the biosynthesis and functions of sterols [29-33], particularly when a targeted gene knockdown system is not yet available for $N$. oceanica IMET1. The chemical inhibitors we employed here have been well studied and the specificities to their corresponding enzymes established [34-38]. 
Table $1 \mathrm{GC} / \mathrm{MS}$ data for sterol profiling from $\mathbf{N}$. oceanica treated with sterol biosynthetic inhibitors

\begin{tabular}{|c|c|c|c|c|c|c|c|c|c|}
\hline \multirow[t]{2}{*}{ Sterols } & \multirow[t]{2}{*}{ Structure } & \multirow[t]{2}{*}{$\mathrm{RT}$ (min) } & \multirow[t]{2}{*}{ RRTC } & \multicolumn{6}{|c|}{ Sterol profile (\%) } \\
\hline & & & & Control & CLO & TBF & TEB & TDM & 25-AZA \\
\hline Squalene & 1 & 10.25 & 0.755 & $-*$ & - & 12.0 & - & - & - \\
\hline Cholesterol & 2 & 13.58 & 1.000 & 74.0 & 70.4 & 61.6 & 62.0 & 56.2 & 81.3 \\
\hline Cholest-8-enol & 3 & 13.75 & 1.012 & - & - & - & - & 8.2 & - \\
\hline Desmosterol & 4 & 13.91 & 1.024 & - & - & - & - & - & 3.0 \\
\hline Pollinastanol & 5 & 14.33 & 1.056 & - & - & - & - & 13.1 & - \\
\hline 4, 14-Dimethylcholest-8-enol & 6 & 14.53 & 1.070 & - & - & - & 1.7 & - & - \\
\hline 24-Methylenecholesterol & 7 & 14.59 & 1.074 & 1.4 & 2.5 & 0.7 & 2.7 & - & 4.0 \\
\hline 24(25)-Dihydrolanosterol & 8 & 15.18 & 1.117 & - & - & - & 1.7 & - & - \\
\hline 4-Methylpollinasterol & 9 & 15.47 & 1.139 & - & - & - & - & 3.7 & - \\
\hline 24-Methylcholesta-5, 25(27)-dienol & 10 & 15.51 & 1.142 & 0.9 & 1.0 & 0.9 & 0.8 & 0.3 & 1.0 \\
\hline Fucosterol & 11 & 15.68 & 1.155 & 10.1 & 10.1 & 10.3 & 7.2 & 6.8 & 6.0 \\
\hline Obtusifoliol & 12 & 15.73 & 1.158 & - & - & - & 0.2 & - & - \\
\hline Isofucosterol & 13 & 15.91 & 1.171 & 13.5 & 16.1 & 14.3 & 8.9 & 9.3 & 4.8 \\
\hline Stigmasta-8, 24(28) E-dienol & 14 & 15.93 & 1.173 & - & - & - & - & 0.4 & - \\
\hline Cycloartenol & 15 & 16.06 & 1.182 & - & - & - & 12.8 & - & - \\
\hline Stigmasta-8, 24(28) Z-dienol & 16 & 16.16 & 1.190 & - & - & - & - & 1.9 & - \\
\hline Cycloartanol & 17 & 16.59 & 1.222 & - & - & - & 2.1 & - & - \\
\hline
\end{tabular}

The inhibitor clomazone (CLO) acts on 1-deoxy-Dxylulose 5-phosphate synthase (DXS), a key regulatory enzyme for chloroplast IPP biosynthesis in higher plants [36]. DXS plays an equivalent role to animal HMGR, which is a key regulatory enzyme for cytosolic IPP biosynthesis via the MVA pathway. CLO had a small effect on sterol profiles (Table 1), but it reduced to $70 \%$ of the total sterol amount of the control, presumably by limiting IPP supplies to the sterol pathway (Figure 2). Terbinafine (TBF) specifically targets squalene epoxidase (SQE) [34], which is the second committed enzyme of the sterol biosynthetic pathway (Figure 2). It is a critical point for inhibition of sterol biosynthesis, as it reduces sterol content but has no direct effect on the biosynthesis of other isoprenoids [34]. TBF-treated cells accumulated a significant amount of squalene (1), supporting the presence and function of SQE (Table 1) and the total amount of sterol was reduced by about $20 \%$. Cells inhibited by tridemorph (TDM), the cycloeucalenol cycloisomerasesterol isomerase (CPI) and sterol C-8 isomerase inhibitor [38], accumulated 9,19-cyclopropyl sterol (pollinastanol, 5) and delta- 8 sterols [obtusifoliol, (12), cholest-8-enol (3), and stigmasta-8,24(28)-dienols (14, 16)] (Table 1). Thus, CPI is required for sterol biosynthesis, implying that $N$. oceanica uses cycloartenol as a precursor in the biosynthesis of other sterols, consistent with the presence of a cycloartenol synthase (CAS) gene (Figure 1). Cycloartenol has been shown to be the signature sterol in almost all photosynthetic organisms [39]. Treatment with tebuconazole (TEB), an inhibitor of the cytochrome P450 CYP51 [37], resulted in accumulation of 4,4,14-trimethyl $(\mathbf{8}, \mathbf{1 5}, \mathbf{1 7})$ and 4,14-dimethyl sterols (6). The relatively large quantities of cycloartanol (17) and cycloartenol (15) (Table 1) further indicate that cycloartenol is the major precursor for the $N$. oceanica sterol biosynthetic pathway (Figure 2). Moreover, the predominance of sterols without side-chain methylation implies that the $14 \alpha$-demethylation step occurs before C-24 methylation and C-4 demethylation (Figure 2). This architecture of the biosynthetic pathway differs from that of land plants and is more similar to the cholesterol biosynthetic pathway in animals and the ergosterol biosynthetic pathway in fungi [40]. 25-azalanosterol (25-AZA) is a specific inhibitor of SMT, which determines sterol compositions [35]. Application of 25-AZA resulted in desmosterol (4) accumulation (Table 1), revealing a similarity to yeast SMT which exhibits a preference for 4,4-desmethylsterols, unlike other algal or plant SMTs [7].

In summary, the collective findings allowed us to propose a sterol biosynthetic pathway in $N$. oceanica 


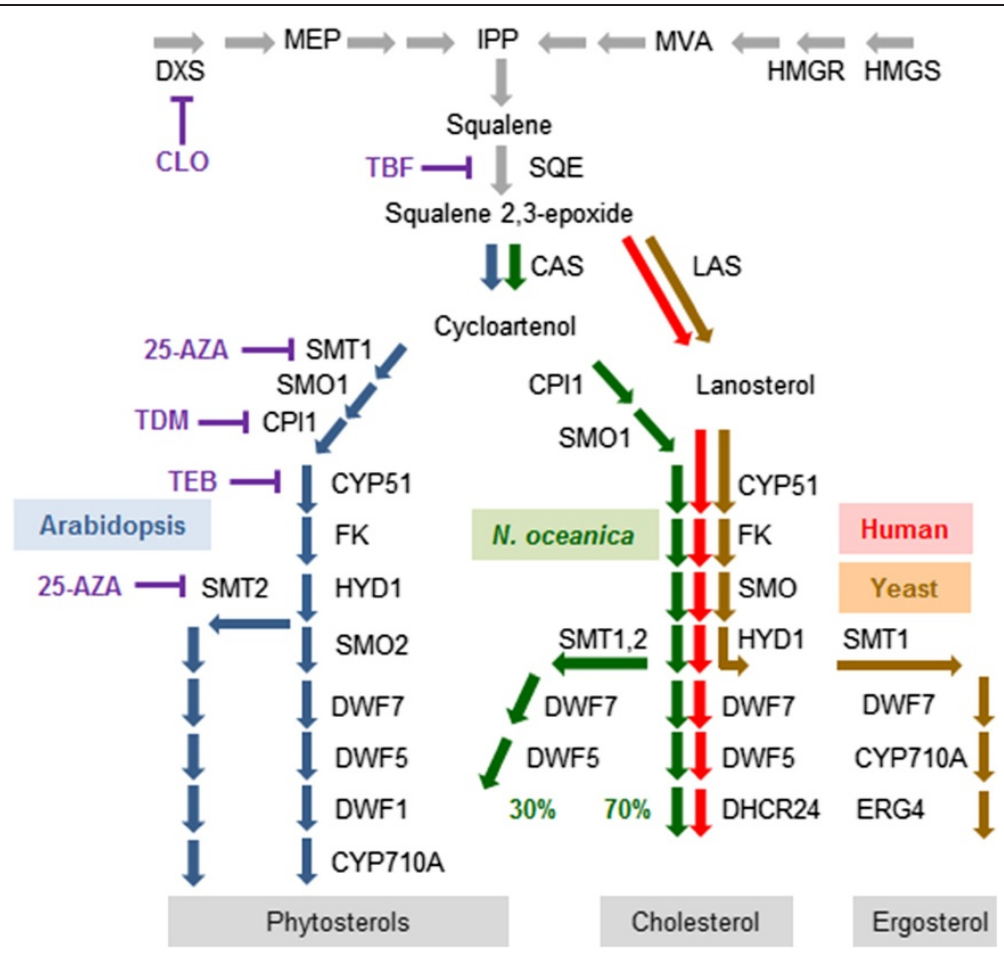

Figure 2 Deduced pathway of sterol biosynthesis in $\mathbf{N}$. oceanica, comparison among plants, humans and yeasts, and sites of action of inhibitors. Enzyme nomenclature is that commonly used in Arabidopsis, because although many human and yeast enzymes have different names and abbreviations, they catalyze equivalent reactions to those of the Arabidopsis enzymes. An exception is Arabidopsis DWF1 (a dual isomerase-reductase), which is absent in Nannochloropsis; instead, this alga has a human-type DHCR24 (the yeast equivalent is ERG4). Full names and functions of enzymes are provided in Additional file 3: Table S1. Lanosterol synthase (LAS) is found in Arabidopsis but has a minor role. Enzyme abbreviations: DXS, 1-deoxy-D-xylulose 5-phosphate synthase; HMGR, hydroxy-methyl-glutaryl-CoA reductase; HMGS, hydroxy-methylglutaryl-CoA synthase; SQE, squalene epoxidase; CAS, cycloartenol synthase; LAS, lanosterol synthase; SMT, sterol methytransferase; SMO, sterol 4-methyl oxidase; CPI, cycloeucalenol cycloisomerase; CYP51, sterol 14-alpha demethylase; FK, sterol C-14 reductase; HYD, sterol C-8 isomerase; DWF7, delta7 sterol C-5 desaturase; DWF5, sterol C-7 reductase; DWF1, sterol C-24(28) isomerase-reductase; CYP710A, sterol C-22 desaturase; DHCR24, dihydrocholesterol reductase; ERG4, sterol delta 24(28) reductase. Abbreviations for metabolites: MEP, methylerythritol phosphate; IPP, isopentenyl pyrophosphate; MVA, mevalonic acid. Abbreviations for inhibitors: CLO, clomazone; TBF, terbinafine; 25-AZA, 25-azalanosterol; TDM, tridemorph; TEB, tebuconazole.

that exhibits both common and distinct features from those in fungi, animals, and green plants (including green algae and land plants) (Figure 2).

\section{The role of sterols in the growth of $N$. oceanica}

To probe the functional roles of sterols in N. oceanica, we next investigated the dynamics of sterol profiles and the expression of their biosynthetic genes during proliferation or cessation of cell division (Figure 3A). Sterol levels exhibited modest increases during the early growth stages of $N$. oceanica cultures, and then increased rapidly at later stages of the culture cycle between $4 \times 10^{7}$ and $10^{8}$ cells ml $^{-1}$ (Figure 3B). The increase of cholesterol accounts for a significant proportion of the increase in total sterols (Figure 3B). Meanwhile, sterol biosynthetic genes showed a coordinated adaptation in the late culture cycle (Figure 3C). All studied genes except DXS and SMT1 were transcriptionally elevated, with maximum levels at the late log phase. Thus, sterol biosynthesis and accumulation appears to be a feature of late cell growth as the culture approaches stationary phase.

Nitrogen depletion is reported to inhibit cell division in $N$. oceanica [41]. To investigate its effects on sterol biosynthesis, we transferred cells to $\mathrm{N}$-replete and $\mathrm{N}$ depleted media for six days. The sterol level in $\mathrm{N}$ depleted cells was about 33\% that of $\mathrm{N}$-replete cells (Additional file 2: Figure S5a). This difference is largely explained by differences in the amount of cholesterol, but surprisingly isofucosterol was disproportionately low, especially compared to fucosterol. This could be explained by differential responses of SMTs to nitrogen depletion. Studies of gene expression during the first $24 \mathrm{~h}$ of nitrogen depletion showed that genes of the MEP pathway were appreciably down-regulated (Additional file 2: Figure S5b), which may explain the subsequent decline in sterol levels. While some genes of sterol biosynthesis were initially up-regulated (for example, $F K$ 


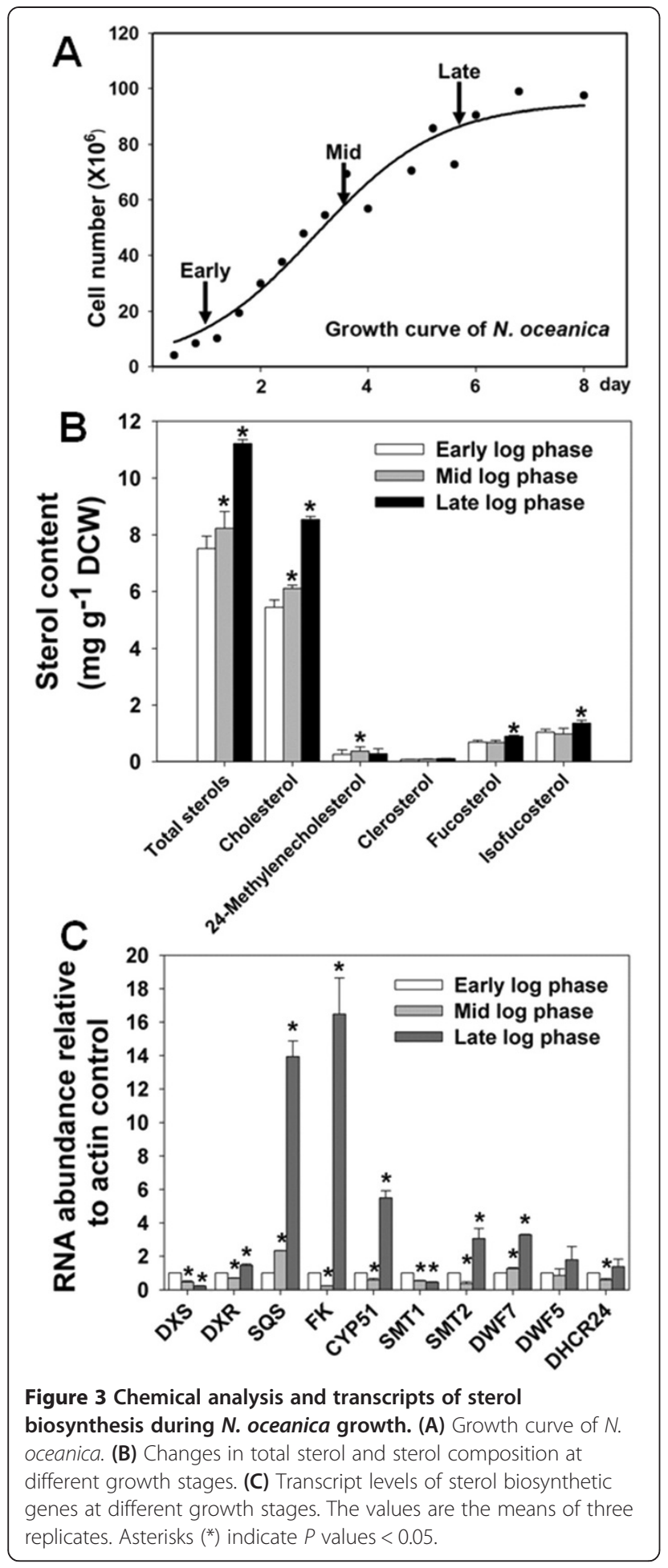

and SMT1), others (for example, SMT2 and DWF5) were rapidly down-regulated (Additional file 2: Figure S5b). These results suggest a complex response to nitrogen depletion but point to a central role for phytosterols, reflecting the importance of SMTs and potentially the methyl donor S-adenosyl methionine.
Effects of light on sterol biosynthesis in $\mathrm{N}$. oceanica

To probe the relationship between sterol biosynthesis and light, we next examined the responses of sterol profiles and their biosynthetic genes to changes in light intensity. Cultures of N. oceanica are typically grown at or below the light saturation point of about $100 \mu \mathrm{mol}$ photons $\mathrm{m}^{-2} \mathrm{~s}^{-1}$, whereas $300 \mu \mathrm{mol}$ photons $\mathrm{m}^{-2} \mathrm{~s}^{-1}$ poses a high light stress. High light is known to cause biochemical damage to the photosynthetic system in higher plants, reducing the efficiency of light utilization.

First, cultures with the same cell density were transferred to constant light intensities of 100 and $300 \mu \mathrm{mol}$ photons $\mathrm{m}^{-2} \mathrm{~s}^{-1}$ for $96 \mathrm{~h}$. RNA and sterols were isolated at the end of the light treatments. The sterol content was lower in cells under $300 \mu \mathrm{mol}$ photons $\mathrm{m}^{-2} \mathrm{~s}^{-1}$ compared with those under $100 \mu \mathrm{mol}$ photons $\mathrm{m}^{-2} \mathrm{~s}^{-1}$ (Figure 4A). This is the result of a significantly reduced cholesterol level, whereas phytosterols (fucosterol and isofucosterol) were significantly increased under $300 \mu \mathrm{mol}$ photons $\mathrm{m}^{-2} \mathrm{~s}^{-1}$ (Figure 4A). This indicates a relationship between light stress and sterol metabolism. All sterol biosynthetic genes examined were expressed at a significantly lower level under $300 \mu \mathrm{mol}$ photons $\mathrm{m}^{-2} \mathrm{~s}^{-1}$ (Figure 4B). Therefore, in response to high light stress, sterol biosynthesis as a whole is repressed, while some specific phytosterols increase in concentration. This observation is consistent with observations that sterol biosynthesis is modified in the high light response of the green alga Dunaliella bardawil [42] and higher plants [43].

To study the short-term effects of high light, cells were first grown under $50 \mu \mathrm{mol}$ photons $\mathrm{m}^{-2} \mathrm{~s}^{-1}$ to mid-log phase, were then darkness-adapted for $12 \mathrm{~h}$ to simulate night, and finally transferred to 100 or $300 \mu \mathrm{mol}$ photons $\mathrm{m}^{-2} \mathrm{~s}^{-1}$ for $12 \mathrm{~h}$. After this time, the photosynthetic efficiency was measured. Samples were collected for analysis of gene expression at the beginning and the end of the 12-h light treatment. The photosynthetic efficiency was significantly higher at $100 \mu \mathrm{mol}$ photons $\mathrm{m}^{-2} \mathrm{~s}^{-1}$ than at $300 \mu \mathrm{mol}$ photons $\mathrm{m}^{-2} \mathrm{~s}^{-1}$ (Figure $4 \mathrm{C}$ ). The transcript levels of all studied genes were also higher at 100 $\mu \mathrm{mol}$ photons $\mathrm{m}^{-2} \mathrm{~s}^{-1}$ than at $300 \mu \mathrm{mol}$ photons $\mathrm{m}^{-2} \mathrm{~s}^{-1}$ (Figure 4D). This further indicates that high light treatment rapidly represses sterol biosynthesis genes and impairs photosynthesis.

To determine if such a repression of gene expression is reversible, cells were treated for $12 \mathrm{~h}$ at $300 \mu \mathrm{mol}$ photons $\mathrm{m}^{-2} \mathrm{~s}^{-1}$ and then transferred to $100 \mu \mathrm{mol}$ photons $\mathrm{m}^{-2} \mathrm{~s}^{-1}$ for $24 \mathrm{~h}$. RNA was isolated at the end of the high light treatment and after 12 and $24 \mathrm{~h}$ adaptation to 100 $\mu \mathrm{mol}$ photons $\mathrm{m}^{-2} \mathrm{~s}^{-1}$, for analysis of gene expression. The results showed that expression of sterol biosynthesis genes increased significantly after $24 \mathrm{~h}$ at $100 \mu \mathrm{mol}$ photons $\mathrm{m}^{-2} \mathrm{~s}^{-1}$ (Figure 4E). Thus, sterol biosynthesis gene expression is highly responsive to such light treatments, 
implying a key role for sterols in adaptation to high light. For comparison, transfer of cells from 100 to $50 \mu \mathrm{mol}$ photons $\mathrm{m}^{-2} \mathrm{~s}^{-1}$ resulted in only minor changes in expression of sterol biosynthesis genes after 12 and $24 \mathrm{~h}$ (Figure 4F), implying that sterol biosynthesis does not play a major role in adaptation to light levels below the saturation point.

\section{Inhibition of sterol biosynthesis in N. oceanica leads to depressed photosynthetic efficiency}

The preceding observations provide evidence of a regulatory role of light in microalgal sterol biosynthesis and the involvement of sterol biosynthesis in photodamage at both the metabolic and gene expression levels. However, whether changes of sterol biosynthesis modulate photosynthesis is still unknown. Thus, we further probed the cellular changes that occurred in response to pharmacological perturbation of sterol biosynthesis.
Cells were grown for $96 \mathrm{~h}$ under $50 \mu \mathrm{mol}$ photons $\mathrm{m}^{-2} \mathrm{~s}^{-1}$ in the presence of $20 \mathrm{mg} \mathrm{l}^{-1}$ CLO, which inhibits DXS activity. This led to a decrease in photosynthetic efficiency (Figure 5A). RBCL is the large subunit of $\mathrm{RuBisCO}$, which incorporates inorganic $\mathrm{CO}_{2}$ into organic forms during photosynthesis [44]. RBCL was transcriptionally depressed following CLO administration (Figure 5B). DXS inhibition led to reduced accumulation of sterols in particular, and of carotenoids and chlorophylls to a much lesser extent (Figure 5C). CLO-treated cells revealed less dense cytoplasm and less distinct organelles. Plastids showed a reduced number of thylakoid membrane structures and a deficiency of normal thylakoid stacking compared with the wild-type cells (Figure 5D,E). This likely explains the decreased photosynthetic efficiency of these cells. Although we showed that sterols were significantly reduced by CLO treatment, determining the contribution of sterols to photosynthetic function required the use of a more

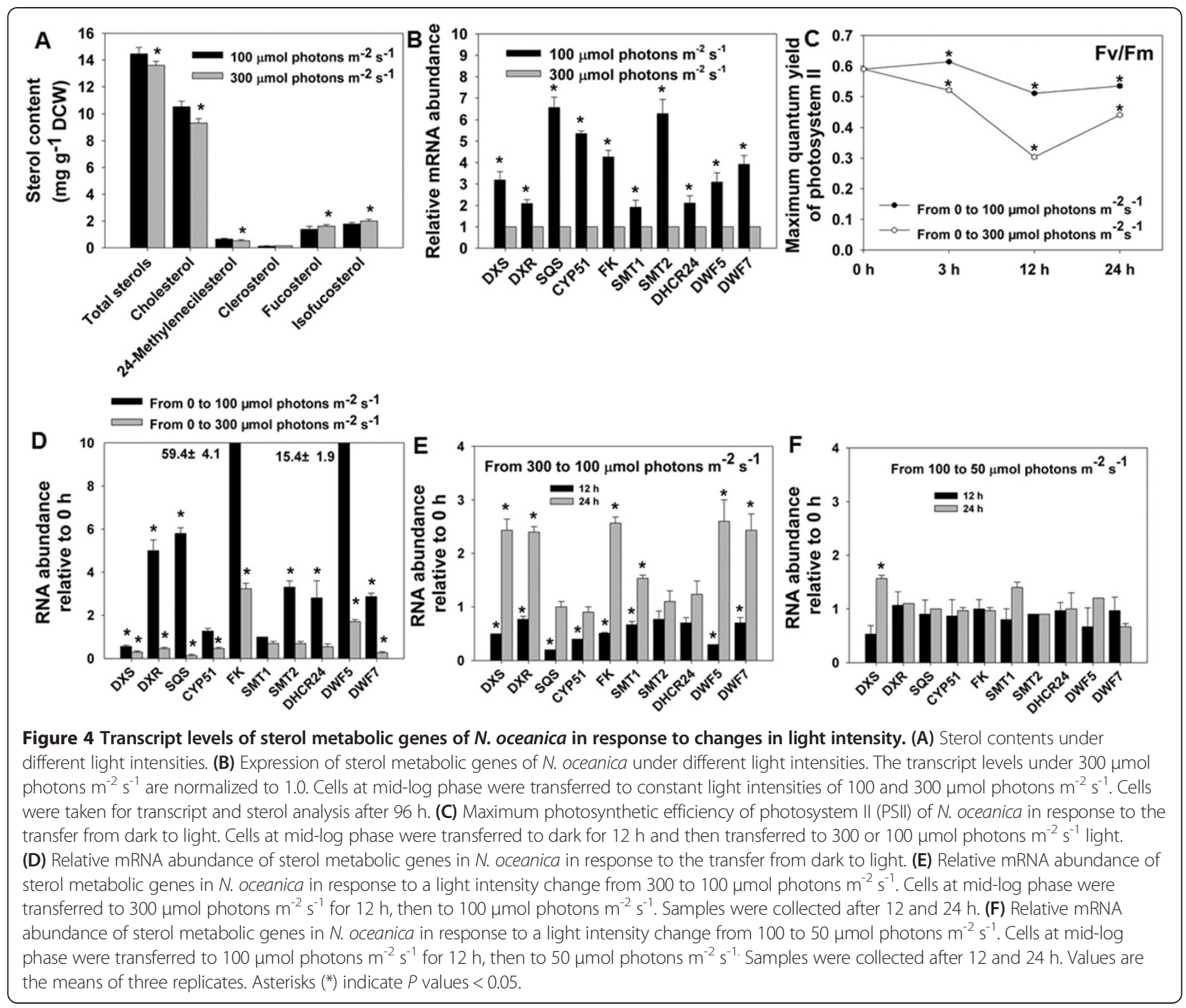


specific inhibitor. Inhibition of the post-squalene sterol biosynthetic pathway by TBF led to a $24 \%$ drop in photosynthetic efficiency relative to the control within $96 \mathrm{~h}$ (Figure 5A). Meanwhile, $R B C L$ was transcriptionally decreased (Figure 5B). TBF-treated cells also contained fewer sterols and more carotenoids and had slightly reduced chlorophyll content (Figure 5C). The cells displayed an aberrant membrane structure with severely affected chloroplasts (Figure 5F). In addition, TBF-treated cells were misshapen compared to untreated cells (Figure 5G,H), implying a defect in membrane structure and function. Therefore, sterols are apparently required for membrane structure, including those of the chloroplast, and this is manifested in reduced photosynthetic function when sterol biosynthesis is inhibited.

\section{Chemical genetic analysis reveals feedback regulation of sterol biosynthesis in $N$. oceanica}

In the $N$. oceanica IMET1 genome, genes for a full MEP pathway for the production of IPP as the building block for isoprenoids were identified (Figure 1). For the MVA pathway, a gene encoding hydroxy-methyl-glutaryl-CoA synthase (HMGS, g249), the first committed enzyme in the MVA pathway, was identified; however, genes encoding enzymes of the remaining steps (including the key regulatory enzyme hydroxy-methyl-glutaryl-CoA reductase; HMGR) were apparently absent (Figure 1). Since the MEP pathway is the sole source of isoprenoids, including sterols, in $N$. oceanica, the control of sterol biosynthesis in the context of IPP biosynthesis is of physiological importance. In animals, cholesterol biosynthesis is highly regulated. Cholesterol over-accumulation leads to decreased biosynthesis of cholesterol and FA, whereas low cholesterol stimulates their synthesis. HMGR, the committed enzyme in isoprenoid and sterol biosynthesis, serves as the primary feedback regulation site to ensure maintenance of lipid homeostasis [45]. Although animal and fungal HMGR is the key target for such regulation, and all such organisms employ an HMGR-binding protein called 'Insig', the signals and molecular mechanisms vary across these species [46]. Higher plants use both MEP and MVA pathways for isoprenoid biosynthesis. Within plant cells, IPP can be exchanged between the cytosol and plastids. Depletion of endogenous sterols by TBF triggers a significant increase in HMGR enzyme activity [34], implying a feedback mechanism in plants similar to that of mammals or fungi. However, the possible regulation of DXS in such a positive feedback regulation is still poorly understood. In higher plants the contribution of IPP from the MVA pathway might attenuate any feedback regulation caused by sterol depletion because of crosstalk between the MEP and MVA pathways. As a result, N. oceanica, which only possesses the MEP pathway, can be an ideal
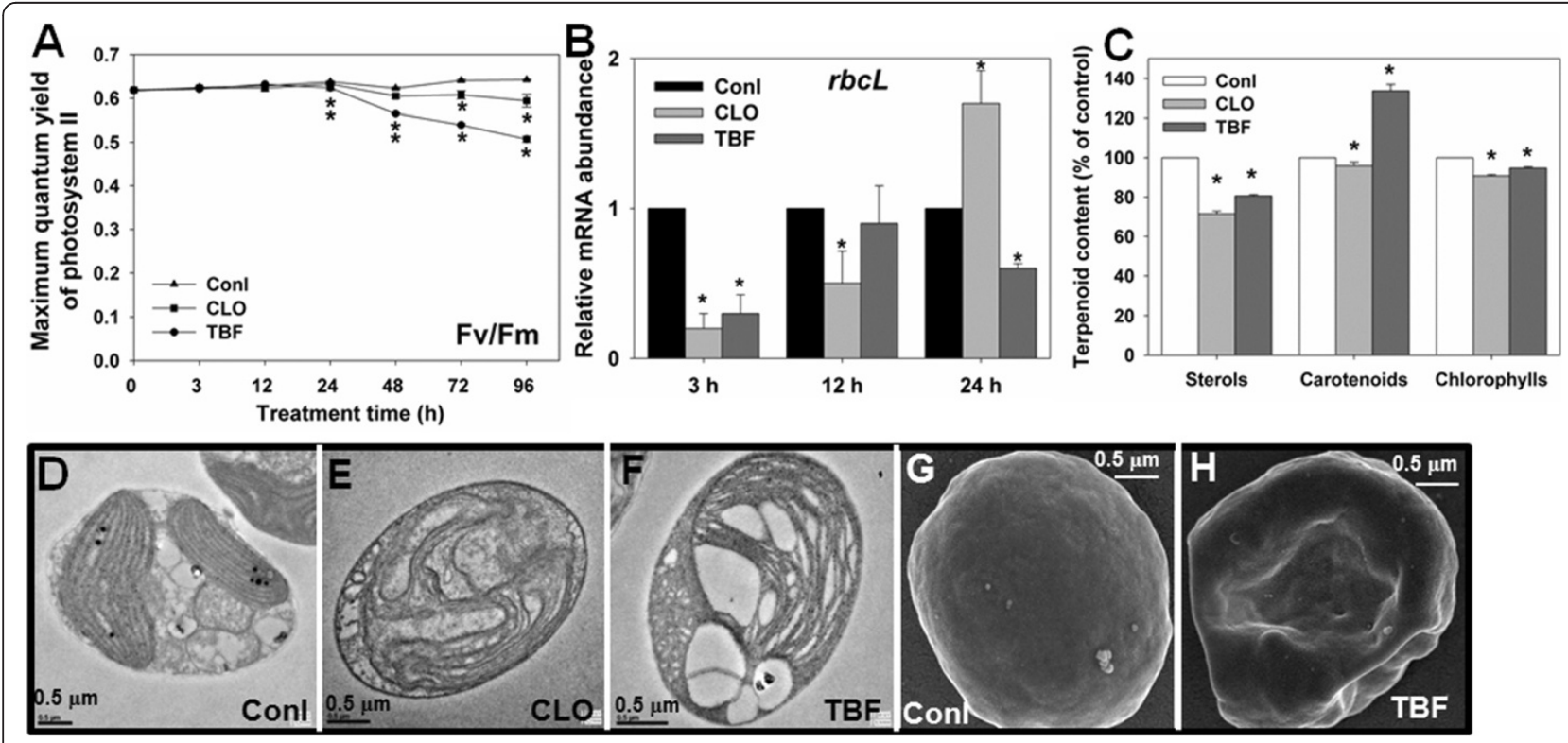

Figure $\mathbf{5}$ The consequence of inhibition of sterol biosynthesis on the photosynthetic activity and apparatus of $\boldsymbol{N}$. oceanica. (A) Effect of CLO (20 mg l-1), and TBF (2.5 mg $\left.\mathrm{I}^{-1}\right)$ on the maximum photosynthetic efficiency of PSII. (B) Transcript levels of RBCL gene of $\mathrm{N}$. oceanica induced by $\mathrm{CLO}\left(20 \mathrm{mg} \mathrm{l}^{-1}\right)$ or TBF $\left(2.5 \mathrm{mg} \mathrm{l}^{-1}\right)$. (C) Levels of sterols, chlorophylls, and carotenoids in the presence of CLO and TBF. Values were normalized (100\% for mock control). (D-F) Transmission electron microscopy analysis of algal cells cultured with DMSO control (D), CLO (E), and TBF (F). (G-H) Scanning electron microscopy analysis of algal cells treated with DMSO control $\mathbf{( G )}$ or TBF (H). Algal cells were grown in medium containing $\mathrm{CLO}$, TBF, or an equivalent amount of DMSO (control) for $96 \mathrm{~h}$ under $50 \mu \mathrm{mol}$ photons $\mathrm{m}^{-2} \mathrm{~s}^{-1}$ light. Scale bars represent $0.5 \mu \mathrm{m}$. Asterisks (*) indicate $P$ values $<0.05$. 
research model for the regulatory role of sterols with respect to DXS. Therefore, we investigated the transcriptional changes of DXS in $N$. oceanica cells in response to sterol depletion caused by CLO and TBF treatments, as well as the cellular response to adding cholesterol to the growth medium.

DXS transcript abundance increased markedly within $48 \mathrm{~h}$ following CLO administration and then decreased, which is a typical inhibition-adaptation response (Figure 6A). The total sterol level was reduced to about $76 \%$ relative to the control after $96 \mathrm{~h}$ of CLO treatment, which can be explained by the reduced supply of IPP due to inhibition of DXS activity (Figure 5C). Moreover, levels of carotenoids and chlorophylls also decreased (Figure 5C), further confirming the low activity of isoprenoid biosynthesis and the committed role of DXS in isoprenoid biosynthesis.

Following TBF application, the total sterol content declined about 20\% (Figure 5C), whereas DXS was transcriptionally induced after $48 \mathrm{~h}$ of treatment (Figure 6B). The induction cannot be explained by the depletion of IPP, since a high level of squalene accumulated in TBF-treated cells (Table 1), suggesting a sufficient supply of IPP for triterpene synthesis. Furthermore, SQS mRNA was reduced to $30 \%$ after $12 \mathrm{~h}$ of treatment (Figure $7 \mathrm{~A}$ ), a result that could potentially be caused by product inhibition by squalene accumulation. On the other hand, carotenoid levels were elevated by $40 \%$ (Figure $5 \mathrm{~B}$ ), and the phytoene synthase gene ( $P S Y$ ) encoding the key committed enzyme step for carotenoid biosynthesis was also transcriptionally up-regulated (Figure 6B). These observations point to stimulated isoprenoid biosynthesis that may be caused by sterol depletion. In addition, the entire MEP pathway and committed cholesterogenic genes were transcriptionally induced by TBF (Figure 7A). Together, the results suggest that TBF-induced sterol starvation stimulates the transcription of DXS and sterol biosynthetic genes.

In the case of sterol accumulation achieved by exogenously added cholesterol, the DXS transcript level was markedly reduced (Figure 6C). Although the carotenoid content did not change, PSY was transcriptionally reduced, supporting the proposal that sterol can act as a feedback regulator to the overall isoprenoid biosynthesis pathway. In summary, the transcription of $D X S$ may be controlled by sterol levels, and sterol biosynthesis potentially exerts feedback regulation on isoprenoid biosynthesis, including cholesterogenesis.

\section{Homeostasis between sterols and lipids in $\mathbf{N}$. oceanica}

Sterol and FA biosynthesis are two major lipid synthetic pathways in eukaryotes, but it is not clear whether they are co-regulated in microalgae. In animals, type I fatty acid synthase (FAS) and other biosynthetic genes are regulated by cellular sterol levels, as are genes that encode important proteins of cholesterol metabolism [13]. To test the link between sterol and FA biosynthesis in $N$. oceanica, the transcript abundances of lipid metabolism genes were investigated following TBF-induced sterol depletion. TBF triggered an increase in type I FAS (g927) transcription after 12 and $24 \mathrm{~h}$ treatment (Figure 7A). This gene was also transcriptionally elevated in cells treated with nitrogen depletion, which is a critical trigger for lipid accumulation (Figure 7B). Consistent with this, the FA level increased over a period of several days following TBF administration (Figure 7C). Next, we analyzed the TAG content after six days of TBF treatment and observed that the TAG concentration was greatly reduced compared to that of the untreated controls (Figure 7D). Therefore, inhibition of sterol biosynthesis by TBF results in an increase in FAs, but these are not accumulated in TAG. This result is supported by thin layer chromatography of FAs (Figure 7D, inset). Simultaneously, the membrane glycerolipids, particularly the photosynthetic membrane lipids such as monogalactosyldiacylglycerol (MGDG), digalactosyldiacylglycerol (DGDG), and phosphatidylglycerol (PG), decreased significantly following TBF treatment (Figure 7E), which can explain the deformed chloroplast and the decreased photosynthetic efficiency of TBF-treated algal cells. Moreover, a remarkable reduction of phosphatidylinositol (PI) and diacylglyceroltrimethylhomoserine (DGTS) was observed (Figure 7E). Taken together, the evidence suggested that the elevated level of total FA in TBF inhibited cells was derived from the increase in de novo FA biosynthesis other than that of storage lipids or membrane lipids. Moreover, free FA should represent a large proportion of the increased amount of total FA.

We therefore propose a feedback system in Nannochloropsis for the regulation of both sterol and FA homeostasis, which is characterized by $(i)$ the induction of sterol and FA biosynthesis by sterol depletion or the inhibition by sterol accumulation and (ii) transcriptional feedback regulation of DXS ensuring maintenance of lipid homeostasis. Interestingly, in animals, HMGR of the MVA pathway is the committed enzyme in isoprenoid and sterol biosynthesis, and serves as the primary site of the feedback regulation [45], suggesting similarity between the algal system and the animal model [17].

\section{Discussion}

\section{Phylogenetic diversity of sterol biosynthetic pathways}

Genomic comparison of evolutionarily tractable algae (Additional file 3: Table S2) revealed the presence of HMGS genes in all of the sampled algal genomes (except Micromonas sp. RCC299 and Ostreococcus tauri which underwent genomic reduction; $[47,48]$ ). (See Figure 1 and Additional file 1). Phylogenetic analysis of HMGS sequences suggests that eukaryotes may have inherited 


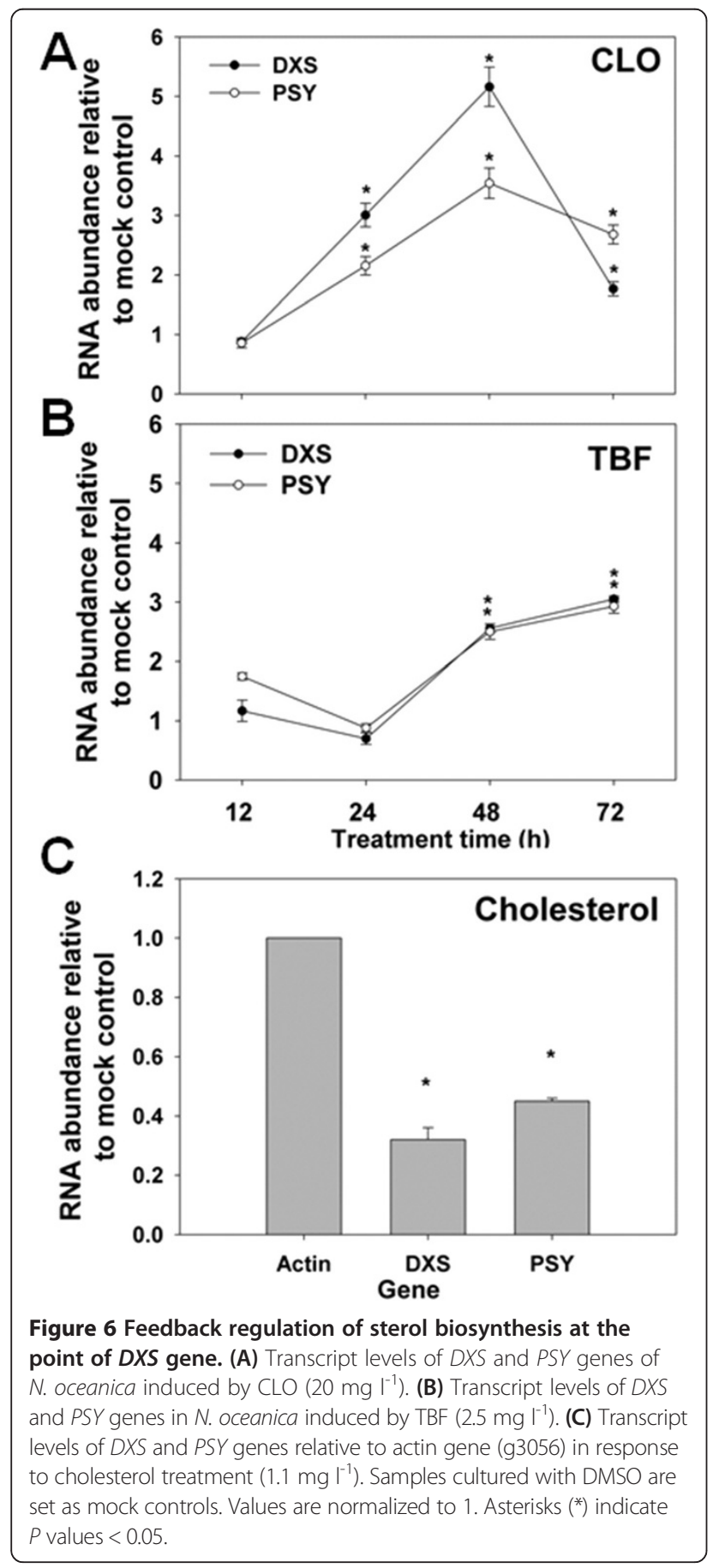

their HMGS genes from Archaea (Additional file 2: Figure S6a). Moreover, purifying selection is apparent, as the nonsynonymous substitution rate is significantly lower than the synonymous substitution rate (Additional file 3: Table S3). On the other hand, HMGS was actively transcribed and regulated in $N$. oceanica under TBF-induced cholesterol depletion (Additional file 2: Figure S6b). In addition, HMGSs from $N$. oceanica and
Ectocarpus siliculosus harbor not only the functional domain of HMGS (PF01154 and PF08540) but also that of beta-ketoacyl synthase (PF00109); see Additional file 3: Table S4. As all remaining enzymes in the MVA pathway are absent in $N$. oceanica, HMGS likely performs a function unrelated to the MVA pathway in this microalga. However, an intermediate form (having four of the five key enzymes of the MVA pathway) was identified in P. tricornutum (Figure 1). Thus, the MVA pathways seem phylogenetically diverse among algal species.

$N$. oceanica produces phytosterols as minor products and cholesterol as the major product; such a sterol profile carries features of both animals and higher plants. Cholesterol and related sterols (ergosterol in yeast and phytosterols in plants) are considered a hallmark of eukaryotes and may even have triggered the evolution of multicellular organisms [49]. In higher plants, cholesterol is normally a minor component of the overall sterol profile ( $1 \%$ to $5 \%)$, and high cholesterol content may have adverse effects on plant growth [50,51]. In some plants, preference in the subcellular distribution of cholesterol was observed, being particularly enriched in chloroplasts and mitochondria and accounting for 24\% of total sterol in each of the organelles (versus 1\% in Phaseolus vulgaris leaves) [52]. N. oceanica, with an extremely high cholesterol content $(>70 \%)$, adds another layer of diversity of sterol biosynthesis and should be an ideal model for studying the functional relationship between cholesterol and photosynthesis.

Moreover, the architecture of the $N$. oceanica sterol biosynthetic pathway also displays both animal and plant features. The underlying mechanisms remained elusive, but evolutionary pressure may have driven the production or elimination of certain sterol structures to adapt to a particular niche. Yeast strains carrying mutations early in the ergosterol pathway exhibit lower survival rates than those with mutations at later parts of the pathway [53]. An SMT2-overexpressing Arabidopsis mutant exhibited an altered campesterol-tositosterol ratio and dramatic phenotypic alterations, whereas a CYP710A1-overexpressing mutant, with an equivalent campesterol-to-stigmasterol ratio, carried a normal phenotype [54]. These results suggest a crucial role of the order of enzymes in the sterol biosynthetic pathway. In addition, $N$. oceanica harbors an animal-signature DHCR24 instead of a plant-type DWF1. Besides the roles in sterol and BR synthesis, DWF1 has a $\mathrm{Ca}^{2+} /$ calmodulin domain related to BR signaling [55]. The domain is absent in animal DHCR24, implying that $N$. oceanica uses a different BR signaling cascade from that of higher plants. Therefore, although the backbone of the sterol biosynthetic pathway appears to be highly conserved in eukaryotic cells, variations in sterol profile and pathway architecture likely have contributed to the distinct morphology. 
A

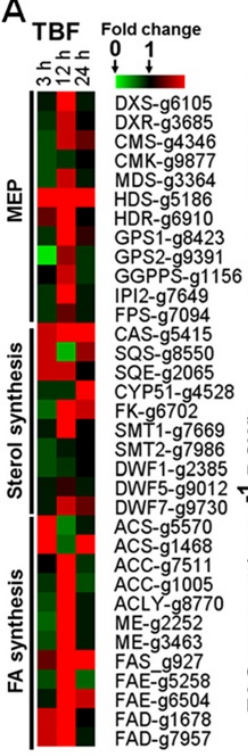

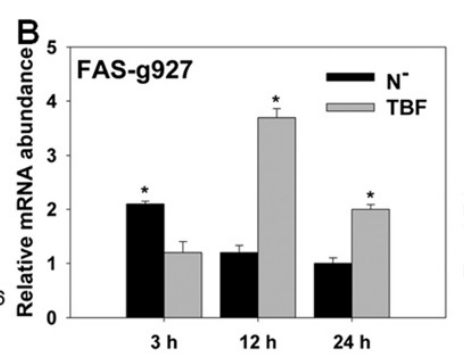

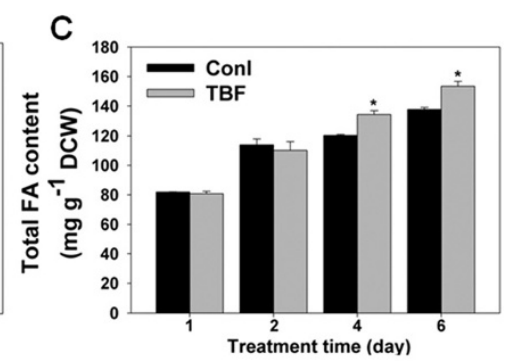

D
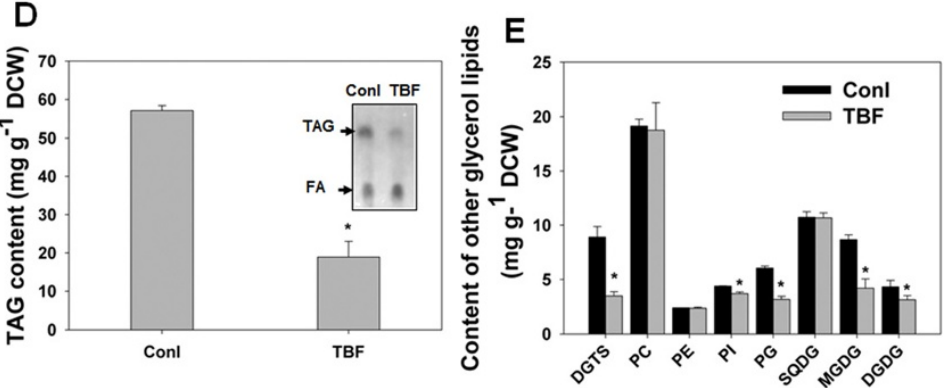

Figure $\mathbf{7}$ Changes in transcripts and lipids of $\boldsymbol{N}$. oceanica in response to sterol starvation induced by TBF. (A) Transcript profiles of cholesterogenic genes and fatty acid (FA) biosynthetic genes in response to TBF $\left(2.5 \mathrm{mg} \mathrm{l}^{-1}\right)$. Red and green indicate up- and down-regulated genes, respectively. (B) Transcript levels of FAS-g927 gene in N. oceanica induced by TBF (2.5 $\left.\mathrm{mg} \mathrm{I}^{-1}\right)$ and nitrogen depletion ( $\left.\mathrm{N}^{-}\right)$. (C) Changes in total FA content induced by TBF. (D) Changes of total TAG content induced by TBF. (E) Changes of glycerol lipid classes (other than TAG) induced by TBF. For all experiments, cultures in exponential phase were treated with TBF ( $2.5 \mathrm{mg} \mathrm{I}^{-1}$ ) or an equivalent amount of DMSO (control). FA content was analyzed at two, four and six days, and glycerolipid content was analyzed at six days. Asterisks $\left({ }^{*}\right)$ indicate $P$ values $<0.05$.

Active involvement of sterol metabolism in $N$. oceanica growth and photosynthesis

The profile of sterols and the transcriptional activity of their biosynthetic genes exhibit dynamic patterns during cell proliferation. Sterols are abundantly synthesized during the late stages of algal cell proliferation, coincident with a period of rapid cell division and membrane synthesis. Defects in the proliferation of cells whose CPI1, CYP51, SQS, and SMT were blocked by specific chemical inhibitors may result from membrane structural defects due to a deficiency in specific sterols. In Arabidopsis, morphological defects in hmg [56], cyp51 [3], and cas1 [2] mutants were shown to be associated with cell wall gaps and aberrant cell wall thickenings. An SMT2-overexpressing Arabidopsis plant, with a higher ratio of campesterol to sitosterol, displayed pleiotropic effects on development, such as reduced growth, increased branching, and low fertility [54]. In addition, sterols transcriptionally activate genes associated with cell expansion and proliferation [12]. Sterol biosynthetic genes are strongly induced at the late-log stage of $N$. oceanica. The inhibition of cell proliferation by the chemical blockage of CPI1, CYP51, SQS, or SMT may be due to defects in sterol biosynthesis and composition.

The inhibition of DXS (responsible for IPP biosynthesis including sterol biosynthesis) and SQE is associated with aberrant photosynthetic efficiency in $N$. oceanica. The defective photosynthesis of DXS-blocked cells may be due to the joint effects of the reduced levels of sterols, carotenoids, and chlorophylls which impair membrane stability as well as light-absorption capacity and management of reactive oxygen species. In contrast, only sterol synthesis is affected in SQE-blocked cells, precluding the possibility that the damage to photosynthesis is caused by a defect of other isoprenoids. Both CLO- and TBF-treated N. oceanica cells display a deformed shape. This appeared to suggest that some of the physiological effects observed were the indirect result of the chemical inhibitor (for example, caused by the well-described toxicity of an increasing pool of free FA in TBF-treated cells). However, specificities of these chemical inhibitors have been confirmed [34-38]; moreover, free FA levels altered in neither CLO-treated (over the full time course of treatment) nor TBF-treated cells (during the first $24 \mathrm{~h}$; despite the decrease of photosynthetic efficiency in the same time frame). It is therefore unlikely that the cell deformation is the only cause for the observed physiological differences, particularly photosynthesis. Furthermore, whereas sterol biosynthetic genes were transcriptionally induced by light, the biosynthesis was reduced upon high light in $N$. oceanica. On the other hand, transcription of SMT was up-regulated upon high light stress in C. reinhardtii [57]. Therefore, sterols play an active role in the response to high light stress in microalgae; moreover, changes in the relative abundance of specific sterols are associated with distinct abnormalities in photosynthesis. 


\section{Feedback regulation of sterol biosynthesis and role of sterols in lipid homeostasis}

The phytosterol biosynthetic pathway leads to production of BRs, which were previously the only known steroid signaling molecules in plants [58]. However, later studies suggest that other sterols besides BRs also function as signaling components, regulating development and gene expression in plants in a manner analogous to the action of cholesterol in mammalian systems [12]. We demonstrated that sterols may also act as signaling molecules in microalgae. In this system, $D X S$ possesses a feedback regulation mechanism in response to sterol starvation. The inhibition of SQE leads to a nearly $20 \%$ decrease of the sterol level, a pronounced inhibitor-mediated increase of the carotenoid level and a remarkable concomitant transcriptional increase of cholesterogenic transcripts. In humans (and other mammals), de novo synthesis of FAs requires cholesterol-mediated regulation of the type I FAS promoter, suggesting that lipogenesis and sterol synthesis are coordinately regulated by cholesterol [59]. There are many genes contributing to FA synthesis, among which type I $F A S$ is one of the most intensively studied. Whereas type I FAS genes appear to be absent in the $C$. reinhardtii genome, an $N$. oceanica IMET1 type I FAS (g927) was transcriptionally up-regulated during triacylglycerol biosynthesis under nitrogen-depletion conditions, suggesting that type I FAS genes may contribute additional FA for the synthesis of TAG in $N$. oceanica [23]. This type I FAS gene was also significantly elevated during the induced depletion of sterol in $N$. oceanica. Moreover, in N. oceanica, during the induced depletion of sterol, the free FA level was stimulated, despite the unknown response of the other FA biosynthetic genes (such as type II FAS genes; [23,25]. Thus, sterols likely control the homeostasis of cholesterol and FA biosynthesis in $N$. oceanica. If so, this would suggest an ancient emergence of the co-regulation of the biosynthesis of FAs and sterols.

\section{Conclusion}

In summary, in microalgae, sterols play essential roles in growth, photosynthesis, and adaption to high light stress. Sterol biosynthesis is potentially feedback-regulated at node of DXS. Sterol and FA metabolisms are co-regulated, such that sterols promote the incorporation of FAs into TAG. Understanding these regulatory mechanisms may lead to rational strategies for improved growth characteristics for $N$. oceanica under challenging environmental conditions and for the development of improved microalgal feedstocks for high-value products or biofuel.

\section{Materials and methods Algal culture}

$N$. oceanica IMET1 was inoculated into modified $\mathrm{f} / 2 \mathrm{li}-$ quid medium, which was prepared with $35 \mathrm{~g} \mathrm{l}^{-1}$ sea salt,
$1 \mathrm{~g} \mathrm{l}^{-1} \mathrm{NaNO}_{3}, 67 \mathrm{mg} \mathrm{l}^{-1} \mathrm{NaH}_{2} \mathrm{PO}_{4}{ }^{*} \mathrm{H}_{2} \mathrm{O}, 3.65 \mathrm{mg} \mathrm{l}^{-1}$ $\mathrm{FeCl}_{3} * 6 \mathrm{H}_{2} \mathrm{O}, 4.37 \mathrm{mg} \mathrm{l}^{-1} \mathrm{Na}_{2}$ EDTA* $2 \mathrm{H}_{2} \mathrm{O}$, trace metal mix $\left(0.0196 \mathrm{mg} \mathrm{l}^{-1} \mathrm{CuSO}_{4}{ }^{*} 5 \mathrm{H}_{2} \mathrm{O}, 0.0126 \mathrm{mg} \mathrm{l}^{-1} \mathrm{NaMoO}_{4}{ }^{*}\right.$ $2 \mathrm{H}_{2} \mathrm{O}, 0.044 \mathrm{mg} \mathrm{l}^{-1} \mathrm{ZnSO}_{4} * 7 \mathrm{H}_{2} \mathrm{O}, 0.01 \mathrm{mg} \mathrm{l}^{-1} \mathrm{CoCl}_{2}$, and $\left.0.36 \mathrm{mg} \mathrm{l}^{-1} \mathrm{MnCl}_{2} * 4 \mathrm{H}_{2} \mathrm{O}\right)$, and vitamin mix $\left(2.5 \mu \mathrm{g} \mathrm{l}^{-1}\right.$ $\mathrm{VB}_{12}, 2.5 \mu \mathrm{g} \mathrm{l}^{-1}$ biotin, and $0.5 \mu \mathrm{g} \mathrm{l}^{-1}$ thiamine $\mathrm{HCl}$ ). The cells were grown in liquid cultures under continuous light (approximately $50 \mu \mathrm{mol}$ photons $\mathrm{m}^{-2} \mathrm{~s}^{-1}$ ) at $25^{\circ} \mathrm{C}$ [23].

Algal cells at certain growth stages were collected for sterol profiling and transcript analysis. Mid-logarithmic phase algal cells were collected and washed with axenic seawater. Equal numbers of cells were re-inoculated in nitrogen-replete medium and nitrogen-deprived medium with $50 \mu \mathrm{mol} \mathrm{m} \mathrm{m}^{-2} \mathrm{~s}^{-1}$ light intensity. Cell aliquots were collected for RNA isolation after being transferred to the designated conditions for 3,12 , and $24 \mathrm{~h}$. Three biological replicates of algal cultures were established for each treatment [23].

Total RNA isolation and quantitative real-time PCR (qPCR) analysis were carried out as previously described [60]. The relative amounts were normalized to the respective beta-actin transcripts. The $2^{-\Delta \Delta C T}$ method was used to analyze qPCR data. Each value in the graphs shows the mean with SE of three experiments. The primer sequences for each gene are given in Additional file 3: Table S5.

\section{Data sources, retrieval, and phylogenetic analyses of the sterol biosynthetic enzymes in algae}

The Arabidopsis protein sequences were retrieved from http://www.arabidopsis.org/. Microalgal genomes were retrieved from the links in Additional file 3: Table S2. Proteins from all genomes were blasted with the Arabidopsis protein queries (Additional file 3: Table S1) using the standalone BLAST software package. The Conserved Domain Database (http://www.ncbi.nlm.nih.gov/cdd/), Pfam (http://pfam.sanger.ac.uk/search), and SMART [61] plus previous genome annotations were employed to confirm the accuracy of chosen sequences. If more than one allele existed, the best-match allele was chosen as representative for the similarity heatmap analysis. The sequences ultimately selected are listed in Additional file 1. Gaps and ambiguously aligned sites were removed using CLUSTALW [62], gBlock [63], and manual validation. ProtTest [64] and PhyML 3.0 [65] were used to select the available model of protein substitution and phylogenetic analyses with a maximum likelihood method. Bootstrap support values were estimated using 100 pseudo-replicates.

\section{Lipid analysis}

Lipids were extracted from lyophilized material according to the method by Yoon [66]. Sterol profiles were analyzed according to our previous description [35]. Lipid class 
analyses were used according to thin layer chromatography (TLC) and quantified using the gas chromatography-fatty acid methyl esters (GC-FAME) method as previously described [66].

\section{Chemical treatments}

Cultures in exponential phase were diluted in fresh media and grown for $12 \mathrm{~h}$. Subsequently, cultures were added with inhibitors at a gradient concentration, or an equivalent amount of DMSO (not to exceed a final concentration of $0.1 \%)$. Cholesterol solution $\left(10 \mathrm{mg} \mathrm{ml}^{-1}\right.$, acetone) was mixed with Tween-20 acetone solution to the designed concentration, and the acetone was removed by a stream of nitrogen. The residue was reconstructed to a water solution and rigid vortex. The solution was sterilized via filtration by passing it through a $0.25-\mu \mathrm{m}$ filter, and it was then added to the culture medium to a desired concentration. Tween-20 without sterol was treated as a blank control. Samples were taken at the indicated periods of time for further analysis.

\section{Analysis of sterols and transcripts related to sterol metabolism}

Cells at mid-log phase were: $(i)$ grown under constant light intensities 100 and $300 \mu \mathrm{mol}$ photons $\mathrm{m}^{-2} \mathrm{~s}^{-1}$. The $96 \mathrm{~h}$ old cells were taken for transcript and sterol profiling analysis; (ii) transferred to darkness for $12 \mathrm{~h}$ and then transferred to 300 or $100 \mu \mathrm{mol}$ photons $\mathrm{m}^{-2} \mathrm{~s}^{-1}$; and samples were collected after $12 \mathrm{~h}$; (iii) cultivated under $300 \mu \mathrm{mol}$ photons $\mathrm{m}^{-2} \mathrm{~s}^{-1}$ for $12 \mathrm{~h}$, and then transferred to $100 \mu \mathrm{mol}$ photons $\mathrm{m}^{-2} \mathrm{~s}^{-1}$; samples were collected after 12 and $24 \mathrm{~h}$; and (iv) cultivated under 100 $\mu \mathrm{mol}$ photons $\mathrm{m}^{-2} \mathrm{~s}^{-1}$ for $12 \mathrm{~h}$, and then transferred to $50 \mu \mathrm{mol}$ photons $\mathrm{m}^{-2} \mathrm{~s}^{-1}$; samples were collected after 12 and $24 \mathrm{~h}$.

\section{Microscopy}

Four-day-old $N$. oceanica cells grown in medium containing CLO (20 mg L $\left.{ }^{-1}\right)$, TBF $\left(2.5 \mathrm{mg} \mathrm{L}^{-1}\right)$, or an equivalent amount of DMSO were sampled for transmission electron microscopy (TEM) [67] and scanning electron microscopy (SEM) analysis [68].

\section{Statistical analysis}

All experiments were replicated at least three times. Data were analyzed using ANOVA followed by paired or unpaired Student's $t$ tests. Differences with $P<0.05$ were considered significant. Asterisks $\left(^{*}\right)$ indicate $P$ values $<0.05$.

\section{Additional files}

Additional file 1: Dataset S1. List of proteins involved in the sterol biosynthetic pathway used to create the similarity heatmap.
Additional file 2: Figure S1. Phylogenetic relationship of organisms studied here. Figure S2. Phylogenetic analysis of the sterol 24(25) isomerase reductase (DWF1) and 24-dehydrocholesterol reductase (DHCR24) using the PhyML 3.0 program. Figure S3. Identification of the sterol compounds extracted from N. oceanica. Figure S4. Inhibition ratio of different isoprenoid biosynthetic inhibitors or sterol biosynthetic inhibitors. Figure S5. Changes in sterol biosynthesis induced by nitrogen depletion. Figure S6. Transcriptional dynamics and phylogenetic analysis of hydroxy-methyl-glutaryl-CoA synthases (HMGS).

Additional file 3: Table S1. List of sterol biosynthetic enzymes in Arabidopsis thaliana. Table S2. Databases used as sequence and annotation sources. Table S3. The ratio of the rate of nonsynonymous substitutions $(K n)$ to the rate of synonymous substitutions $(K S)$ of hydroxy-methyl-glutaryl-CoA synthase (HMGS). Table S4. Comparison of sequence identity and conserved domains of hydroxy-methyl-glutarylCoA synthase (HMGS) proteins between algal species and Arabidopsis thaliana. Table S5. Primers used in this study.

\section{Abbreviations}

25-AZA: 25-azalanosterol; BR: brassinosteroid; CAS: cycloartenol synthase; CLO: clomazone; CPI: cycloeucalenol cycloisomerase; CYP51: sterol 14-alpha demethylase; CYP710A: sterol C-22 desaturase; DGDG: digalactosyldiacylglycerol; DGTS: diacylglyceroltrimethylhomoserine; DHCR24: dihydrocholesterol reductase; DWF1: sterol C-24(28) isomerase-reductase; DWF5: sterol C-7 reductase; DWF7: delta7 sterol C-5 desaturase; DXS: 1-deoxy-D-xylulose 5phosphate synthase; ERG4: sterol delta 24(28) reductase; FA: fatty acid; FAS: fatty acid synthase; FK: sterol C-14 reductase; GC-FAME: gas chromatography-fatty acid methyl esters; HMGR: hydroxy-methyl-glutaryl-CoA reductase; HMGS: hydroxy-methyl-glutaryl-CoA synthase; HYD: sterol C-8 isomerase; IPP: isopentenyl pyrophosphate; Kn: nonsynonymous substitutions; Ks: synonymous substitutions; LAS: Ianosterol synthase; MEP: methyl erythritol phosphate; MGDG: monogalactosyldiacylglycerol; MVA: mevalonic acid; PG: phosphatidyl glycerol; PI: phosphatidylinositol; PSY: phytoene synthase; qPCR: quantitative real-time PCR; SBI: sterol biosynthetic inhibitor; SEM: scanning electron microscopy; SMO: sterol 4-methyl oxidase; SMT: sterol methyltransferase; SQE: squalene epoxidase; SREBP: sterol regulatory element binding protein; TAG: triacylglycerol; TBF: terbinafine; TDM: tridemorph; TEB: tebuconazole; TEM: transmission electron microscopy; TLC: thin layer chromatography.

\section{Competing interests}

The authors declare that they have no competing interests.

\section{Authors' contributions}

$Y L$ and $W Z$ planned the experiments. $Y L, W Z, W L, J L, J$, and $F L$ performed the experiments. $Y L$ and $W Z$ analyzed the data. $Y L, W Z, S S$, and JX wrote the paper. All authors read and approved the final manuscript.

\section{Acknowledgements}

This work was supported by the Natural Science Foundation of China (31200063), the Ministry of Science and Technology of China (2012CB721101), the Basic Research in Science and Technology Program of Qingdao (13-1-4-229-jch) and the Knowledge Innovation Program of the Chinese Academy of Sciences (KSZD-EW-Z-017-2).

\section{One-Sentence Summary}

Cholesterol is the major sterol of an oleaginous unicellular alga Nannochloropsis oceanica, and its biosynthesis is tightly linked to fatty acid synthesis and correspondingly, the production of triacylglycerol.

Received: 5 March 2014 Accepted: 1 May 2014

Published: 30 May 2014

\section{References}

1. Nes WD: Biosynthesis of cholesterol and other sterols. Chem Rev 2011, 111:6423-6451.

2. Babiychuk E, Bouvier-Navé $P$, Compagnon V, Suzuki M, Muranaka T, Van Montagu M, Kushnir S, Schaller H: Allelic mutant series reveal distinct functions for Arabidopsis cycloartenol synthase 1 in cell viability and plastid biogenesis. Proc Natl Acad Sci 2008, 105:3163-3168. 
3. Kim HB, Lee $H$, Oh CJ, Lee $H-Y$, Eum HL, Kim H-S, Hong Y-P, Lee $Y$, Choe $S$, An CS, Choi S-B: Postembryonic seedling lethality in the sterol-deficient Arabidopsis cyp51A2 mutant is partially mediated by the composite action of ethylene and reactive oxygen species. Plant Physiol 2010 152:192-205.

4. Smagghe G: Ecdysone, Structures and Functions. New York: Springer; 2009

5. Auger CJ, Coss D, Auger AP, Forbes-Lorman RM: Epigenetic control of vasopressin expression is maintained by steroid hormones in the adult male rat brain. Proc Natl Acad Sci 2011, 108:4242-4247.

6. Santner A, Calderon-Villalobos LIA, Estelle M: Plant hormones are versatile chemical regulators of plant growth. Nat Chem Biol 2009, 5:301-307.

7. Miller MB, Haubrich BA, Wang Q, Snell WJ, Nes WD: Evolutionarily conserved $\Delta 25$ (27)-olefin ergosterol biosynthesis pathway in the alga Chlamydomonas reinhardtii. J Lipid Res 2012, 53:1636-1645.

8. Eisenreich W, Rohdich F, Bacher A: Deoxyxylulose phosphate pathway to terpenoids. Trends Plant Sci 2001, 6:78-84.

9. Vickers CE, Gershenzon J, Lerdau MT, Loreto F: A unified mechanism of action for volatile isoprenoids in plant abiotic stress. Nat Chem Biol 2009 5:283-291.

10. Posé D, Castanedo I, Borsani O, Nieto B, Rosado A, Taconnat L, Ferrer A, Dolan L, Valpuesta V, Botella MA: Identification of the Arabidopsis dry2/ sqe 1-5 mutant reveals a central role for sterols in drought tolerance and regulation of reactive oxygen species. Plant J 2009, 59:63-76.

11. Clouse SD: Arabidopsis mutants reveal multiple roles for sterols in plant development. Plant Cell 2002, 14:1995-2000.

12. He JX, Fujioka S, Li TC, Kang SG, Seto H, Takatsuto S, Yoshida S, Jang JC: Sterols regulate development and gene expression in Arabidopsis. Plant Physiol 2003, 131:1258-1269.

13. Bennett MK, Lopez JM, Sanchez HB, Osborne TF: Sterol regulation of fatty acid synthase promoter: coordinate feedback regulation of two major lipid pathways. J Biol Chem 1995, 270:25578-25583.

14. Bengoechea-Alonso MT, Ericsson J: SREBP in signal transduction: cholesterol metabolism and beyond. Curr Opin Cell Biol 2007, 19:215-222

15. Osborne TF: Cholesterol homeostasis: Clipping out a slippery regulator. Curr Biol 1997, 7:R172-R174.

16. Horton J, Goldstein J, Brown M: SREBPs: transcriptional mediators of lipid homeostasis. In Cold Spring Harbor Symposia on Quantitative Biology. Woodbury, NY: Cold Spring Harbor Laboratory Press; 2002:491-498.

17. Horton JD: SREBPs: activators of the complete program of cholesterol and fatty acid synthesis in the liver. J Clin Invest 2002, 109:1125-1131.

18. Wijffels RH, Barbosa MJ: An outlook on microalgal biofuels. Science 2010, 329:796-799.

19. Georgianna DR, Mayfield SP: Exploiting diversity and synthetic biology for the production of algal biofuels. Nature 2012, 488:329-335.

20. Volkman J: Sterols in microorganisms. Appl Microbiol Biotechnol 2003, 60:495-506.

21. Desmond E, Gribaldo S: Phylogenomics of sterol synthesis: insights into the origin, evolution, and diversity of a key eukaryotic feature. Genome Biol Evol 2009, 1:364-381.

22. Véron B, Billard C, Dauguet JC, Hartmann MA: Sterol composition of Phaeodactylum tricornutum as influenced by growth temperature and light spectral quality. Lipids 1996, 31:989-994.

23. Li J, Han D, Wang D, Ning K, Hu Q, Xu J: Choreography of transcriptomes and lipidomes of Nannochloropsis reveals the mechanisms of oleaginousness in microalgae. Plant Cell 2014, http://dx.doi.org/10.1105/ tpc.113.121418.

24. Wei $L$, Xin $Y$, Wang DM, Jing XY, Zhou Q, Su XQ, Jia J, Ning K, Chen F, Hu Q, Xu $\mathrm{J}$ : Nannochloropsis plastid and mitochondrial phylogenomes reveal organelle diversification mechanism and intragenus phylotyping strategy in microalgae. BMC Genomics 2013, 14:534. doi:10.1186/1471-2164-14-534.

25. Vieler A, Wu G, Tsai CH, Bullard B, Cornish AJ, Harvey C, Reca IB, Thornburg C, Achawanantakun R, Buehl CJ, et al: Genome, functional gene annotation, and nuclear transformation of the heterokont oleaginous alga Nannochloropsis oceanica CCMP1779. Plos Genet 2012, 8:e1003064.

26. Radakovits $R$, Jinkerson RE, Fuerstenberg SI, Tae H, Settlage RE, Boore JL, Posewitz MC: Draft genome sequence and genetic transformation of the oleaginous alga Nannochloropis gaditana. Nat Comm 2012, doi:10.1038/ ncomms3356.

27. Wang D, Ning K, Li J, Hu Q, Xu J: Nannochloropsis genomes reveal evolution of microalgal oleaginous traits. Plos Genet 2014, doi:10.1371/ journal.pgen.1004094.
28. King N, Westbrook MJ, Young SL, Kuo A, Abedin M, Chapman J, Fairclough S, Hellsten U, Isogai Y, Letunic I, et al: The genome of the choanoflagellate Monosiga brevicollis and the origin of metazoans. Nature 2008, 451:783-788.

29. Blackwell HE, Zhao Y: Chemical genetic approaches to plant biology. Plant Physiol 2003, 133:448-455.

30. Girke T, Cheng L-C, Raikhel N: ChemMine. A compound mining database for chemical genomics. Plant Physiol 2005, 138:573-577.

31. Hicks GR, Raikhel NV: Opportunities and challenges in plant chemical biology. Nat Chem Biol 2009, 5:268-272.

32. Stockwell BR: Chemical genetics: ligand-based discovery of gene function. Nat Rev Genet 2000, 1:116-125.

33. Walsh DP, Chang Y-T: Chemical genetics. Chem Rev 2006, 106:2476-2530.

34. Wentzinger LF, Bach TJ, Hartmann MA: Inhibition of squalene synthase and squalene epoxidase in tobacco cells triggers an up-regulation of 3hydroxy-3-methylglutaryl coenzyme A reductase. Plant Physiol 2002, 130:334-346.

35. Zhou W, Cross GAM, Nes WD: Cholesterol import fails to prevent catalystbased inhibition of ergosterol synthesis and cell proliferation of Trypanosoma brucei. J Lipid Res 2007, 48:665-673.

36. Mueller C, Schwender J, Zeidler J, Lichtenthaler H: Properties and inhibition of the first two enzymes of the non-mevalonate pathway of isoprenoid biosynthesis. Biochem Soc Trans 2000, 28:792-793.

37. Lamb DC, Kelly DE, Manning NJ, Hollomon DW, Kelly SL: Expression, purification, reconstitution and inhibition of Ustilago maydis sterol 14ademethylase (CYP51; P45014DM). FEMS Microbiol Lett 1998, 169:369-373.

38. Bladocha M, Benveniste P: Manipulation by Tridemorph, a systemic fungicide, of the sterol composition of maize leaves and roots. Plant Physiol 1983, 71:756-762

39. Režen T, Debeljak N, Kordiš D, Rozman D: New aspects on lanosterol 14ademethylase and cytochrome P450 evolution: lanosterol/cycloartenol diversification and lateral transfer. J Mol Evol 2004, 59:51-58.

40. Kim HB, Schaller H, Goh CH, Kwon M, Choe S, An CS, Durst F, Feldmann KA, Feyereisen R: Arabidopsis cyp51 mutant shows postembryonic seedling lethality associated with lack of membrane integrity. Plant Physiol 2005, 138:2033-2047.

41. Dong H-P, Williams E, Wang D-Z, Xie Z-X, Hsia R-C, Jenck A, Halden R, Li J, Chen F, Place AR: Responses of Nannochloropsis oceanica IMET1 to long-term nitrogen starvation and recovery. Plant Physiol 2013, 162:1110-1126.

42. Zelazny AM, Shaish A, Pick U: Plasma membrane sterols are essential for sensing osmotic changes in the halotolerant alga Dunaliella. Plant Physiol 1995, 109:1395-1403.

43. Huang L-S, Grunwald C: Sterol and phospholipid changes during alfalfa seed germination. Phytochemistry 1988, 27:2049-2053.

44. Johnson X, Wostrikoff K, Finazzi G, Kuras R, Schwarz C, Bujaldon S, Nickelsen J, Stern DB, Wollman F-A, Vallon O: MRL1, a conserved pentatricopeptide repeat protein, is required for stabilization of rbcL $\mathrm{mRNA}$ in Chlamydomonas and Arabidopsis. Plant Cell 2010, 22:234-248.

45. Brown MS, Goldstein JL: Cholesterol feedback: from Schoenheimer's bottle to Scap's MELADL. J Lipid Res 2009, 50:S15-S27.

46. Burg JS, Espenshade PJ: Regulation of HMG-CoA reductase in mammals and yeast. Progr Lipid Res 2011, 50:403-410.

47. Worden AZ, Lee J-H, Mock T, Rouzé P, Simmons MP, Aerts AL, Allen AE, Cuvelier ML, Derelle E, Everett MV, et al: Green evolution and dynamic adaptations revealed by genomes of the marine picoeukaryotes Micromonas. Science 2009, 324:268-272.

48. Palenik B, Grimwood J, Aerts A, Rouzé P, Salamov A, Putnam N, Dupont C, Jorgensen R, Derelle E, Rombauts $S$, et al: The tiny eukaryote Ostreococcus provides genomic insights into the paradox of plankton speciation. Proc Nat Acad Sci 2007, 104:7705-7710.

49. Brown AJ, Galea AM: Cholesterol as an evolutionary response to living with oxygen. Evolution 2010, 64:2179-2183.

50. Diener AC, Li H, Zhou W, Whoriskey WJ, Nes WD, Fink GR: Sterol methyltransferase 1 controls the level of cholesterol in plants. Plant Cell 2000, 12:853-870.

51. Carland F, Fujioka S, Nelson T: The sterol methyltransferases SMT1, SMT2 and SMT3 influence Arabidopsis development through nonbrassinosteroid products. Plant Physiol 2010, 153:741-756.

52. Brandt RD, Benveniste $P$ : Isolation and identification of sterols from subcellular fractions of bean leaves (Phaseolus vulgaris). Biochim Biophys Acta (BBA) - Biomembr 1972, 282:85-92. 
53. Dupont $\mathrm{S}$, Lemetais $\mathrm{G}$, Ferreira T, Cayot $\mathrm{P}$, Gervais $\mathrm{P}$, Beney L: Ergosterol biosynthesis: a fungal pathway for life on land? Evolution 2012, 66:2961-2968

54. Schaeffer A, Bronner R, Benveniste P, Schaller H: The ratio of campesterol to sitosterol that modulates growth in Arabidopsis is controlled by STEROL METHYLTRANSFERASE 2;1. Plant J 2001, 25:605-615.

55. Du L, Poovaiah BW: $\mathrm{Ca}^{2+} /$ calmodulin is critical for brassinosteroid biosynthesis and plant growth. Nature 2005, 437:741-745.

56. Suzuki M, Nakagawa S, Kamide Y, Kobayashi K, Ohyama K, Hashinokuchi H, Kiuchi R, Saito K, Muranaka T, Nagata N: Complete blockage of the mevalonate pathway results in male gametophyte lethality. $J$ Exp Bot 2009, 60:2055-2064.

57. Ledford HK, Baroli I, Shin JW, Fischer BB, Eggen RIL, Niyogi KK: Comparative profiling of lipid-soluble antioxidants and transcripts reveals two phases of photo-oxidative stress in a xanthophyll-deficient mutant of Chlamydomonas reinhardtii. Mol Genet Genom 2004, 272:470-479.

58. Hartmann M-A: Plant sterols and the membrane environment. Trends Plant Sci 1998, 3:170-175.

59. Xiong S, Chirala SS, Wakil SJ: Sterol regulation of human fatty acid synthase promoter I requires nuclear factor-Y- and Sp-1-binding sites. Proc Nat Acad Sci 2000, 97:3948-3953.

60. Lu Y, Chi X, Li Z, Yang Q, Li F, Liu S, Gan Q, Qin S: Isolation and characterization of a stress-dependent plastidial $\Delta 12$ fatty acid desaturase from the Antarctic microalga Chlorella vulgaris NJ-7. Lipids 2010, 45:179-187.

61. Letunic I, Doerks T, Bork P: SMART 7: recent updates to the protein domain annotation resource. Nucleic Acids Res 2012, 40:D302-D305.

62. Chenna R, Sugawara H, Koike T, Lopez R, Gibson TJ, Higgins DG, Thompson JD: Multiple sequence alignment with the Clustal series of programs. Nucleic Acids Res 2003, 31:3497-3500.

63. Talavera G, Castresana J: Improvement of phylogenies after removing divergent and ambiguously aligned blocks from protein sequence alignments. Syst Biol 2007, 56:564-577.

64. Darriba D, Taboada GL, Doallo R, Posada D: ProtTest 3: fast selection of best-fit models of protein evolution. Bioinformatics 2011, 27:1164-1165.

65. Guindon S, Dufayard J-F, Lefort V, Anisimova M, Hordijk W, Gascuel O: New algorithms and methods to estimate maximum-likelihood phylogenies: assessing the performance of PhyML 3.0. Syst Biol 2010, 59:307-321.

66. Yoon K, Han D, Li Y, Sommerfeld M, Hu Q: Phospholipid:diacylglycerol acyltransferase is a multifunctional enzyme involved in membrane lipid turnover and degradation while synthesizing triacylglycerol in the unicellular green microalga Chlamydomonas reinhardtii. Plant Cell 2012, 24:3708-3724.

67. Schrick K, Fujioka S, Takatsuto S, Stierhof Y-D, Stransky H, Yoshida S, Jürgens $\mathrm{G}$ : A link between sterol biosynthesis, the cell wall, and cellulose in Arabidopsis. Plant J 2004, 38:227-243.

68. Aarts MG, Keijzer CJ, Stiekema WJ, Pereira A: Molecular characterization of the CER1 gene of Arabidopsis involved in epicuticular wax biosynthesis and pollen fertility. Plant Cell 1995, 7:2115-2127.

doi:10.1186/1754-6834-7-81

Cite this article as: Lu et al:: Regulation of the cholesterol biosynthetic pathway and its integration with fatty acid biosynthesis in the oleaginous microalga Nannochloropsis oceanica. Biotechnology for Biofuels 2014 7:81.

\section{Submit your next manuscript to BioMed Central and take full advantage of:}

- Convenient online submission

- Thorough peer review

- No space constraints or color figure charges

- Immediate publication on acceptance

- Inclusion in PubMed, CAS, Scopus and Google Scholar

- Research which is freely available for redistribution 